Table

Adjusted cognitive function tests scores at year 25: the CARDIA study, 1985-2010

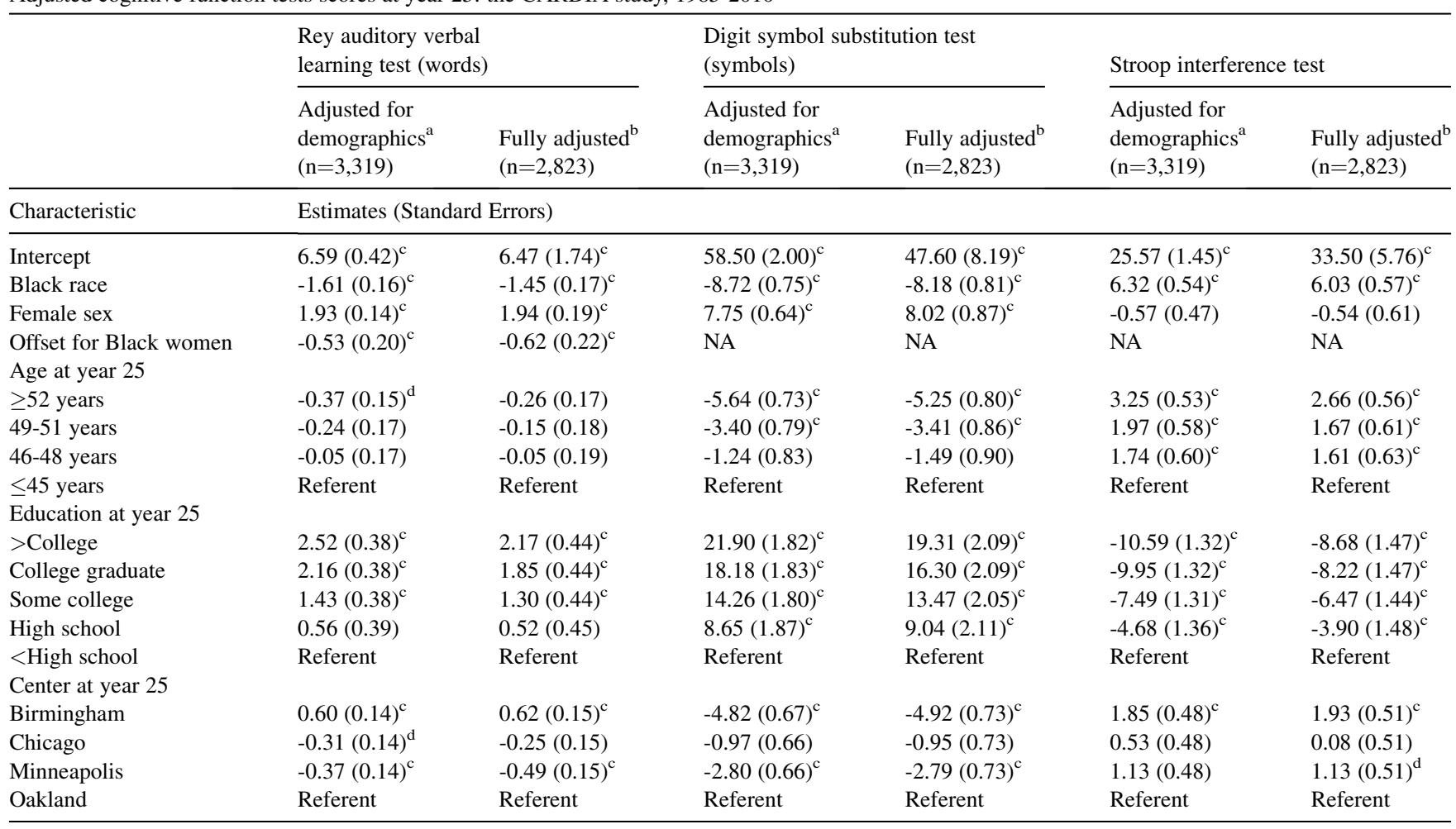

Interpretative Key: For the Rey auditory verbal learning test, the range of scores is 0-15, with increasing scores indicating better performance. For the Digit Symbol Substitution Test, the range of scores is 0-133, with increasing scores indicating better performance. For the Stroop interference test, a higher score indicates worse performance on the task. NA $=$ not applicable.

${ }^{a}$ Model adjusted for race, sex, age, educational attainment at year 25, and center at year 25.

${ }^{\mathrm{b}}$ Model adjusted for race, sex, age, educational attainment at year 25, clinical center at year 25, and year 10 values of depressive symptoms, systolic and diastolic blood pressure, fasting glucose, diabetes status, height, physical activity, waist circumference, and cigarette smoking.

${ }^{\mathrm{c}} \mathrm{P} \leq 0.01$

${ }^{\mathrm{d}} \mathrm{P} \leq 0.05$

Results: Cognitive scores varied by sex, race, education, and age. For all tests, cognitive scores were lower in blacks than whites, after adjusting for demographics (Table). Compared to men, w omen scored (mean \pm standard error, SE) $7.75 \pm 0.64$ symbols higher on the DSST $(\mathrm{P}<0.001)$, but similarly on the Stroop $(\mathrm{P}=0.22)$. A small race-by-sex interaction $(\mathrm{P}=0.01)$ was seen for the RAVLT, but not for the other 2 tests. Adjusted mean RAVLT scores $( \pm \mathrm{SE})$ were $10.08 \pm 0.09$ for white women, $8.15 \pm$ 0.10 for white men, $7.94 \pm 0.10$ for black women, and $6.54 \pm 0.12$ for black men. Cognitive scores tended to be lower in older adults and those with fewer years of education. Most demographic differences persisted after full adjustment, although the magnitude of the age and sex differences reduced slightly (Table). Results were similar in analyses including APOE e4. Conclusions: Demographic differences in cognition appear in mid-life and are not fully explained by clinical or lifestyle factors.

\section{P2-304 APPLICATION OF SYSTEMS MEDICINE TO ALZHEIMER'S DISEASE AND DEMENTIA RESEARCH: A SYSTEMATIC REVIEW}

Elzbieta Kuzma ${ }^{1}$, Declan G. Bates ${ }^{2}$, Iain A. Lang ${ }^{3}$, Cédric Annweiler ${ }^{4}$, Olivier Beauchet ${ }^{4}$, David Bennett ${ }^{5}$, Kenneth Langa ${ }^{6}$, Robert Wallace ${ }^{7}$, Kristine Yaffe ${ }^{8}$, David J. Llewellyn ${ }^{1},{ }^{1}$ University of Exeter Medical School, Exeter, United Kingdom; ${ }^{2}$ University of Warwick, Coventry, United Kingdom; ${ }^{3}$ University of Exeter Medical School / National Institute for Health Research Collaboration for Leadership in Applied Health Research and Care South West Peninsula, Exeter, United Kingdom; ${ }^{4}$ Angers University Hospital, Angers, France; ${ }^{5}$ Rush University Medical Center,
Chicago, Illinois, United States; ${ }^{6}$ University of Michigan, Ann Arbor, Michigan, United States; ${ }^{7}$ University of Iowa, Iowa City, Iowa, United States; ${ }^{8}$ University of California San Francisco, San Francisco, California, United States.Contact e-mail: e.kuzma@exeter.ac.uk

Background: Systems medicine offers a novel paradigm that is well established in the study of complex chronic diseases that arise as the result of multiple interacting factors such as cancer and diabetes. Systems medicine models may help to account for the dynamic interactions between biological, clinical and environmental factors in Alzheimer's disease (AD) and dementia. Our aim was to examine the extent to which systems medicine has been applied to dementia research. Methods: We conducted a systematic review using EMBASE, Medline and PsycInfo from inception to January 2014 plus backward and forward citation searches of included publications following a pre-defined protocol (see Figure). Experts in the field were contacted to identify any additional relevant publications. We searched for publications applying a computational or mathematical model of $\mathrm{AD}$ or dementia and integrating dynamically interacting factors on multiple levels (biological, clinical and/or environmental). No date or language restrictions were used. Two reviewers independently screened titles and abstracts, and conducted full text reviews. Discrepancies were resolved by discussion with involvement of two additional reviewers. Results: We selected 24 studies for full-text review. Two studies met all inclusion criteria and provide useful examples to illustrate the potential of the systems medicine paradigm. The first study incorporated a multifactorial computational model of AD pathogenesis, and was subsequently extended in the second study to incorporate the hypothesized role of estrogen in 
amyloid-beta regulation. They demonstrated that treatments directed at multiple targets may be more effective than single-target therapies, and make several experimentally testable predictions. These models took into account the dynamic interactions between biological and clinical factors, although the number of clinical factors was highly limited. Conclusions: Systems medicine has not yet been widely applied in the field of dementia research. However, the two computational models identified illustrate the potential of this paradigm to conceptualize dementia in a more realistic fashion. Further research is necessary to develop and validate models that incorporate a wider range of dynamically interacting biological, clinical and environmental factors. Ultimately systems medicine may provide the basis for more targeted multidrug therapies and complex interventions.

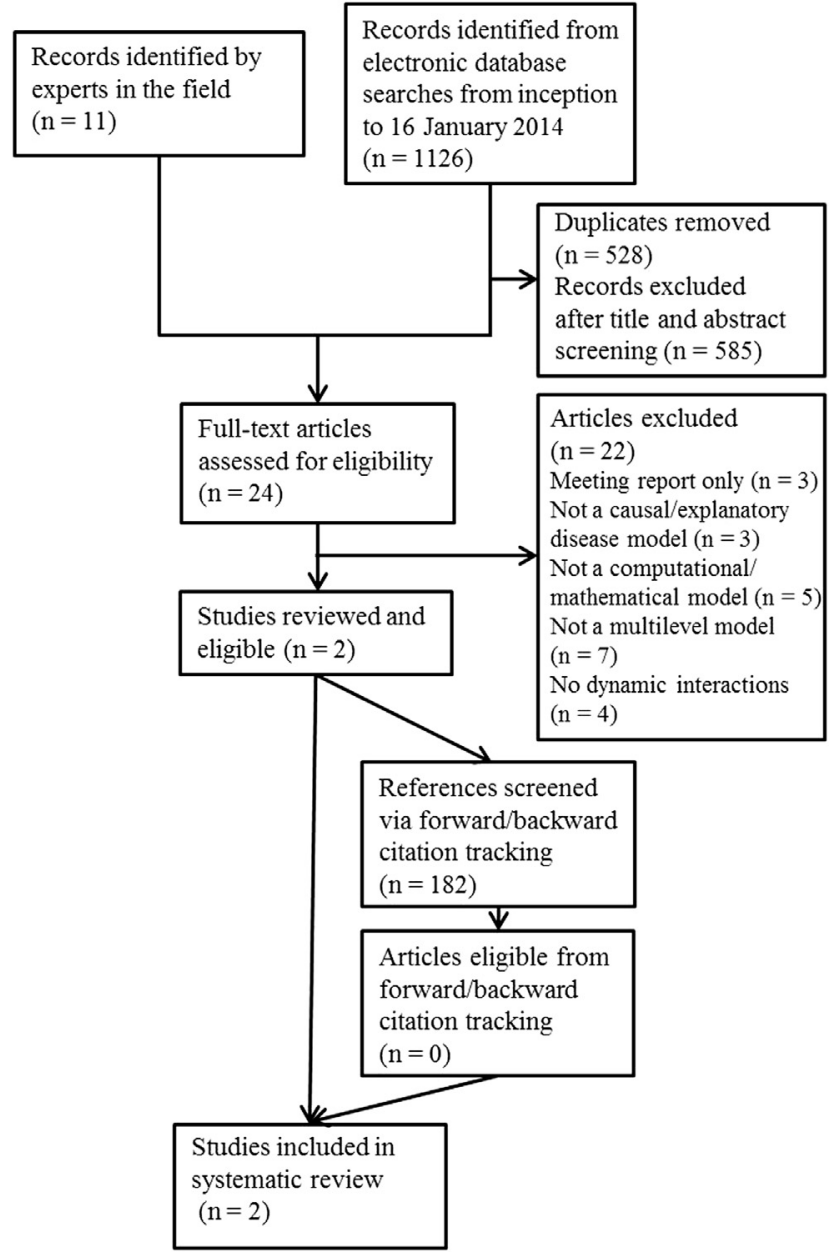

P2-305 ASSOCIATION BETWEEN REDUCED KIDNEY FUNCTION AND COGNITIVE PERFORMANCE IN THE OLDEST OLD: THE HEALTH AND ANEMIA STUDY

Emma Riva ${ }^{1}$, Sara Mandelli ${ }^{2}$, Mauro Tettamanti ${ }^{3}$, Angela Recchia ${ }^{3}$, Ugo Lucca ${ }^{1},{ }^{1}$ IRCCS-Istituto di Ricerche Farmacologiche Mario Negri, Milan, Italy; ${ }^{2}$ IRCCS-Istituto di Ricerche Farmacologiche Mario Negri, Milan, Italy; ${ }^{3}$ IRCCS-Istituto di Ricerche Farmacologiche Mario Negri, Milano, Italy.Contact e-mail: riva@marionegri.it

Background: Both glomerular filtration rate (GFR) and cognitive performance decline with age. However, the association between renal function and cognition has been poorly examined in the oldest old. Objective: to investigate the relationship between reduced renal function and cognitive performance in the oldest old in a population-based study of 85-years or older residents in the municipality of Biella, Italy (Health and Anemia Study). Methods: GFR was estimated (eGFR) using the Modification of Diet in Renal Disease (MDRD) equation. Cognitive performance was assessed with a battery of neuropsychological tests: the Mini-Mental State Examination, CERAD Word List Memory, CERAD Word List Recall, CERAD Word List Recognition, Visual Search on Matrices of Digits and the Short Stroop Colour-Word. Results: Data on serum creatinine concentration and neuropsychological tests were available for 557 participants (mean age: 89.2 years; mean educational level: 6.8 years; women: $71.8 \%$ ). Of these oldest old, $98(17.6 \%)$ had an eGFR $<45 \mathrm{~mL} / \mathrm{min} / 1.73 \mathrm{~m} 2,17$ of whom $(3.1 \%)$ had an eGFR $<30 \mathrm{~mL} / \mathrm{min} / 1.73 \mathrm{~m}$ 2. Subjects with an eGFR $<45 \mathrm{~mL} / \mathrm{min} /$ $1.73 \mathrm{~m} 2$ had lower mean scores on all neuropsychological tests compared to those with an eGFR $\geq 60 \mathrm{~mL} / \mathrm{min} / 1.73 \mathrm{~m} 2$, but differences between groups reached marginal statistical significance only for Visual Search on Matrices of Digits (age, sex and education adjusted $\mathrm{p}=0.0421$, Bonferroni corrected). Conclusions: In the present population-based study, moderately-severely reduced kidney function was generally not associated with a significant reduction in cognitive performance. The possible effect of kidney impairment on selective and sustained attention should be investigated in larger numbers of oldest old with moderately or severely reduced GFR.

\section{P2-306 “CAT: CENTENARI A TRIESTE”: A STUDY FOR A CLINICAL-BIOLOGICAL AND PSYCHO-SOCIAL DATABASE OF THE CENTENARIAN POPULATION IN TRIESTE}

Gabriella Marcon ${ }^{1}$, Ugo Lucca ${ }^{2}$, Nicola Montano ${ }^{3}$, Alessandro Pincherle ${ }^{4}$, Adele Maggiore $^{5}$, Dino Trento ${ }^{5}$, Sonia Ammesso ${ }^{6}$, Mauro Tettamanti ${ }^{7}$, ${ }^{1}$ University of Udine, Udine, Italy; ${ }^{2}$ Istituto di Ricerche Farmacologiche Mario Negri, Milano, Italy; ${ }^{3}$ Università degli Studi di Milano, Milano, Italy; ${ }^{4}$ IRCCS Istituto Neurologico Besta, Milano, Italy; ${ }^{5}$ ASS1, Trieste, Italy; ${ }^{6}$ IRCCS Istituto di Ricerche Farmacologiche Mario Negri, Milano, Italy; ${ }^{7}$ IRCCS-Istituto di Ricerche Farmacologiche Mario Negri, Milano, Italy. Contact e-mail: gabriella.marcon@uniud.it

Background: The continued expansion of the elderly population and a growing awareness of age-related disorders have prompted considerable interest in the study of the individuals who reach 100 years (longevity outliers). This peculiar group represents the fastest growing age group worldwide. Part of them are still self-sufficient, a condition defined "successful aging". In Italy, the prevalence of centenarians is $1-2 / 10,000$ inhabitants, but in some areas the number is higher, such as in Barbagia (Sardinia) or in Trieste, city in which the prevalence of centenarians reaches 6-8/10,000 inhabitants. Trieste, as result of its particular geographical position and history, is a melting pot of cultures and religions, the opposite of a founder population as in Sardinia. For this reason, we have started to collect past and present data on the psychobiologic and socio-demographic characteristics related to the centenarians living in Trieste to create a database. Methods: In Trieste city (211,000 inhabitants) there are about 150 centenarians, a large part of them still self-sufficient. After signed consent, the centenarians are enrolled in the study to collect all data available on past and present habits and lifestyle, education, nutrition, physical activity, social life, state of health and pathological conditions and lifetime history of drug intake. Moreover, a neuropsychological examination is performed in addition to the sleep recording and the Heart Rate Variability evaluation for vegetative nervous system study. Blood, stool, urine and saliva samples are collected. The project, which started in January 2014, is implemented by a network of Family Doctors, neuropsychologists, nurses, health care operators of ASS1 Triestina and the relatives of the centenarians. Results: Our goal is to create a database of the centenarians living in Trieste and, in the future, a centenarian register. Basal characteristics of the subjects are reported. Conclusions: The database will be useful not only for studying habits and lifestyle, health conditions and socio-demographic characteristics of this peculiar population, but also for genetic and biological research. The study demonstrates the possibility 